\title{
RG Fixed Points in Supergravity Duals of 4-d Field Theory and Asymptotically AdS Spaces
}

\author{
M. Porrati and A. Starinets由 \\ Theory Division CERN, Ch 1211 Geneva 23, Switzerland \\ and \\ Department of Physics, NYU, 4 Washington Pl., New York, NY 10003, USA
}

\begin{abstract}
Recently, it has been conjectured that supergravity solutions with two asymptotically $A d S_{5}$ regions describe the RG flow of a 4-d field theory from a UV fixed point to an interacting IR fixed point. In this paper we lend support to this conjecture by showing that, in the UV (IR) limit, the two-point function of a minimally coupled scalar field depends only on the UV (IR) region of the metric, asymptotic to $A d S_{5}$. This result is consistent with the interpretation of the radial coordinate of Anti de Sitter space as an energy scale, and it may provide an analog of the Callan-Symanzik equation for supergravity duals of strongly coupled field theories.
\end{abstract}

CERN-TH/99-49

February 1999

\footnotetext{
${ }^{1}$ e-mail: massimo.porrati@nyu.edu, andrei.starinets@physics.nyu.edu
} 
The duality between gauge theories and (super)string geometries, first proposed for conformal field theories [1, 2, 3], also holds in a more general setting. Particularly interesting is the case when the superstring geometry is only asymptotically $A d S_{5}$. This setting describes 4-d gauge theories that are (super)conformal only in the ultraviolet. In the infrared, they may confine and/or screen charges [4, 5, 6], or reduce to another conformal field theory [7, 8, 9]. The 5-d metric that describes the latter case has two regions, respectively "far" and "close" to the brane, where the metric is asymptotically $A d S_{5}$. The interpolating metric is still invariant under the 4-d Poincaré group and it reads [8]:

$$
d s^{2}=e^{2 \phi(z)}\left(d z^{2}+\eta_{\mu \nu} d x^{\mu} d x^{\nu}\right), \quad \eta_{\mu \nu}=\operatorname{diag}(-1,1,1,1) .
$$

For small $z$ ("far" from the brane) the prefactor in the metric has the following expansion:

$$
\phi(z)=-\log \left(\frac{z}{R_{U V}}\right)+O(z)
$$

Here, $R_{U V}$ is the radius of the "far" AdS region. For large $z$ the expansion is, instead:

$$
\phi(z)=-\log \left(\frac{z}{R_{I R}}\right)+O(1 / z) .
$$

In ref. [8] it was shown that an interpolating metric as in Eq. (11) exists, and that it describes mass deformations of $\mathrm{N}=4 S U(N)$ super-Yang Mills theory. The metric was shown to exist using some general properties of type IIB, 5-d, gauged supergravity [10, 11]. The proof given in ref. [8] does not rely heavily on specific properties of gauged supergravity, and it is valid also in a more general context; in type 0 strings, for instance.

The interpolating metric was interpreted as describing the renormalization group flow from an UV N=4 superconformal theory to an IR conformal theory. This interpretation is suggested by the UV/IR connection [5, 12, 13], i.e. by the identification of the $A d S_{5}$ coordinate, $z$, with an appropriate length scale in the 4 -d field theory.

A problem with a direct interpretation of the equations of motion of gauged supergravity as RG equations is that they are second order, instead of first order. This seems to suggest that $z$ cannot be identified with the renormalization scale of the 4-d field theory. Purpose of this paper is to prove that this identification is nevertheless correct, namely, that the IR physics of the boundary field theory is essentially independent of $z$. This will be proven by finding an analog of the Callan-Symanzik equation of field theory, that describes the change of the two-point function of a composite operators under change of the UV cutoff. This equation will show, that the low-momentum limit of the two-point function is only sensitive to the IR region of the 5-d geometry -i.e. the region close to the brane. We will also show that the high-momentum limit of the two-point function is sensitive only to the UV region of the 5-d geometry. 
Let us start by recalling that in the geometry dual of field theory the two-point function of a composite operator, $\mathcal{O}\left(x^{\mu}\right)$, with source $\psi\left(x^{\mu}\right)$ is found as follows?

1. The field $\psi$ is promoted to a 5 -d field $\psi\left(x^{\mu}, z\right)$. It obeys some boundary conditions, given in refs. [2, 3]. Here we find it convenient to follow the prescription of ref. [2], and to choose as boundary conditions at small $z$ a plane 4 -d wave: $\psi\left(x^{\mu}\right)=\exp \left(i k_{\mu} x^{\mu}\right)$.

$$
\psi\left(x^{\mu}, z\right)=e^{i k_{\mu} x^{\mu}} \psi_{k}(z),\left.\quad \psi_{k}(z)\right|_{z=\epsilon}=1, \quad \lim _{z \rightarrow \infty} \psi_{k}(z)=0, \quad k_{\mu} k^{\mu} \equiv k^{2}>0
$$

A few comments are in order here. a) Here $\epsilon$ is an UV regulator, and must be chosen much smaller than any other length scale in the problem, in particular, $k \epsilon \ll 1$. b) When $k^{2}<0$ the boundary condition at large $z$ is $\psi_{k}(z) \propto \exp \left(i|k| z+i k_{\mu} x^{\mu}\right)$. c) If the 5 -d geometry is not $A d S_{5}$ at large $z$, but rather it develops a singularity at finite $z=a$, then the boundary condition at $a$ is that $\psi_{k}(z)$ is regular near $z=a$. In this paper, we set aside the latter possibility, and assume that the large- $z$ geometry of the 5-d space obeys Eq. (3).

2. The 5-d field $\psi_{k}(z)$ obeys free scalar equation of motion:

$$
\left[-\partial_{z} \partial_{z}-3 \phi_{z}(z) \partial_{z}+k^{2}+e^{2 \phi(z)} M^{2}(z)\right] \psi_{k}(z)=0 .
$$

Here, $\phi_{z}(z) \equiv \partial_{z} \phi(z)$. The square-mass term $M^{2}(z)$ becomes constant both in the IR and in the UV

$$
\lim _{z \rightarrow 0} M^{2}(z)=M_{U V}^{2}, \quad \lim _{z \rightarrow \infty} M^{2}(z)=M_{I R}^{2} .
$$

Generically, $M_{U V}^{2} \neq M_{I R}^{2}$.

3. Finally, the two-point function $A\left(k^{2}\right)=\int d^{4} x \exp \left(i k_{\mu} x^{\mu}\right)\langle\mathcal{O}(x) \mathcal{O}(0)\rangle$ is given by [2]

$$
A\left(k^{2}\right)=\left[e^{3 \phi(z)} \psi_{k}^{*}(z) \partial_{z} \psi_{k}(z)\right]_{\epsilon}^{\infty} \equiv\left[e^{3 \phi(z)} \frac{\partial_{z} \psi_{k}(z)}{\psi_{k}(z)}\right]_{\epsilon}^{\infty} .
$$

The latter form of $A\left(k^{2}\right)$ is independent of the normalization condition at $z=\epsilon$, and it is valid whenever $\psi_{k}(z)$ obeys the correct boundary condition at $z=\infty$.

We want to prove, first of all, that the low-momentum behavior of $A\left(k^{2}\right)$ is insensitive to the small- $z$ region of the 5 -d geometry.

The key to the proof is a first-order equation for the two-point function, somewhat reminiscent of the Callan-Symanzik equation. Using Eq. (5), and defining

$$
A\left(k^{2}, z\right)=-e^{3 \phi(z)} \partial_{z} \log \left[\psi_{k}(z)\right],
$$

\footnotetext{
${ }^{2}$ Here, for simplicity, we will restrict ourselves to minimally-coupled scalar fields.
} 
one finds the equation:

$$
\partial_{z} A\left(k^{2}, z\right)=e^{-3 \phi(z)} A^{2}\left(k^{2}, z\right)-k^{2} e^{3 \phi(z)}-M^{2}(z) e^{5 \phi(z)}
$$

Notice that the boundary conditions at large $z$ ensure that $\lim _{z \rightarrow \infty} A\left(k^{2}, z\right)=0$. The two-point function plays the role of the initial condition for Eq. (9):

$$
A\left(k^{2}, \epsilon\right)=A\left(k^{2}\right)
$$

Notice that, analogously to the Callan-Symanzik equation, Eq. (9) does not fix the value of the two-point function: that comes from solving the second-order equation Eq. (5), subject to the boundary conditions given in Eq. (4). Eq. (9) describes instead the evolution of a quantity, $A\left(k^{2}, z\right)$ that coincides with the true two-point function at small $z$.

It is tempting to interpret $A\left(k^{2}, z\right)$ as the two-point function computed with a cutoff $z$. This is indeed true if for low momenta, $k \rightarrow 0, A\left(k^{2}, z\right)$ differs from $A\left(k^{2}\right)$ by at most a multiplicative factor, and an additive factor either polynomial in $k^{2}$ or of higher order in the $k^{2}$ expansion:

$$
A\left(k^{2}, z\right)=Z^{2}(z) A\left(k^{2}\right)+P\left(k^{2}, z\right)+O\left[k^{2} z^{2} A\left(k^{2}\right)\right], \quad k z \ll 1
$$

The multiplicative factor $Z^{2}(z)$ is interpreted as the wave-function renormalization of the operator $\mathcal{O}\left(x^{\mu}\right)$. The polynomial $P\left(k^{2}, z\right)$ changes only the contact terms in the two-point function, without affecting its behavior at non-coincident points. The last term is negligible in the infrared limit.

Notice that, whenever Eq. (11) holds, the dependence of the two-point function on the UV geometry, i.e. the small- $z$ region, is completely factored into contact terms and a wave-function renormalization constant. In field theory, the same can be said verbatim for the dependence of the two-point function on the UV cutoff. Therefore, if Eq. (11) holds, the coordinate $z$ plays exactly the role of a length cutoff. This is another manifestation of the UV/IR connection for non-conformal theories. More interestingly, Eq. (11) says that in geometries with two AdS regions, as in the examples in refs. [8, 9], the infrared behavior of the 4-d theory is completely described by the IR $A d S_{5}$ geometry, given in Eqs. (1,3). To study the IR, one can ignore the behavior of the metric in the UV region $k z>1$. As a concrete application of this result, the quantity $M_{I R}^{2}$ is related to the IR scaling dimension of $\mathcal{O}, \Delta_{I R}$, by the standard AdS formula [2, 3, 14]

$$
\Delta_{I R}=2+\sqrt{4+M_{I R}^{2} R_{I R}^{2}}
$$

Eq. (11) is easily proven. It is sufficient to notice that $A\left(k^{2}, z\right)$ is a smooth function of the initial conditions $A\left(k^{2}\right)$. This is a standard property of ordinary differerential equations with smooth coefficients, as Eq. (11). A proof of this theorem can be found in [15. 
We are interested in the $k$-dependence of $A\left(k^{2}, z\right)$. Smoothness in the initial conditions (and $z$ ) implies $A\left(k^{2}, z\right)=F\left(A\left(k^{2}\right), z, k^{2}\right)$, with $F\left(A, z, k^{2}\right)$ a smooth function of $A, z$ and $k^{2}$. The field-theory interpretation of $A\left(k^{2}\right)$ tells us that the small- $k^{2}$ expansion of $A\left(k^{2}\right)$ reads 3

$$
A\left(k^{2}\right)=A(0)+Q\left(k^{2}\right)+c k^{2 \Delta_{I R}-4}+O\left(k^{2 \Delta_{I R}-2}\right) .
$$

Here $c$ is a nonzero constant, positive by unitarity of the 4-d IR theory; $Q\left(k^{2}\right)$ is a polynomial in $k^{2}$, vanishing at $k^{2}=0$.

By expanding $A\left(k^{2}, z\right)$ near the initial condition $A(0)$ we find

$$
\begin{aligned}
A\left(k^{2}, z\right)= & F\left(A(0), z, k^{2}\right)+\left.\frac{\partial F}{\partial A}\left(A, z, k^{2}\right)\right|_{A=A(0)}[A-A(0)]+O\left\{[A-A(0)]^{2}\right\} \\
= & F(A(0), z, 0)+R\left(k^{2}, z\right)+\left.\frac{\partial F}{\partial A}(A, z, 0)\right|_{A=A(0)}[A-A(0)]+ \\
& +O\left\{[A-A(0)]^{2},[A-A(0)]^{2} k^{2}\right\}
\end{aligned}
$$

where $R\left(k^{2}, z\right)$ is a polynomial in $k^{2}$, vanishing at $k^{2}=0$. Eq. (11), with $Z^{2}=\partial F /\left.\partial A\right|_{A=A(0)}$, follows immediately from the expansion in Eq. (13) and the smoothness of $F\left(A, z, k^{2}\right)$.

Positivity of the wave-function renormalization $Z$ is proven as follows. By analytic continuation in $k^{2}, A\left(k^{2}, z\right)$ becomes an analytic function with a cut along the real negative axis. By its definition, given in Eq. (8), it obeys $A\left(k^{2 *}, z\right)=A^{*}\left(k^{2}, z\right)$. By splitting Eq. (9) into real and imaginary part, we find the equation

$$
\partial_{z} \operatorname{Im} A\left(k^{2}, z\right)=2 e^{-3 \phi(z)} \operatorname{Re} A\left(k^{2}, z\right) \operatorname{Im} A\left(k^{2}, z\right)
$$

Expanding its solution near $k^{2}=0$ we find f thanks to Eq. (13):

$$
\operatorname{Im} A\left(k^{2}, z\right)=e^{2 \int_{\epsilon}^{z} e^{-3 \phi(w)} \operatorname{Re} A(0, w) d w} c \sin \left(4 \Delta_{I R} \pi\right) k^{2 \Delta_{I R}-4}+O\left(k^{2 \Delta_{I R}-2}\right) .
$$

From this equation, it follows that the wave-function renormalization factor is positive:

$$
Z(z)=e^{\int_{\epsilon}^{z} e^{-3 \phi(w)} \operatorname{Re} A(0, w) d w} .
$$

After having studied the IR limit of the two-point function, we want to study the opposite limit, namely $k^{2} \rightarrow \infty$. We want to show that, in this limit, $A\left(k^{2}\right)$ can be computed by approximating the metric (11) with its UV AdS form, given in Eq. (2). We shall do so by writing Eq. (5) in the form of an integral equation and studying its "Jost solution" in the limit $k^{2} \rightarrow \infty$.

\footnotetext{
${ }^{3}$ Here we write the expression valid for generic, non-integer $\Delta_{I R}$. For integer $\Delta_{I R}$, the non-analytic term reads $k^{2\left(\Delta_{I R}-2\right)} \log k^{2}$.

${ }^{4}$ Again, we write an equation valid for non-integer $\Delta_{I R}$. It is trivial to see that the equation for $\Delta_{I R}$ integer gives the same result for the $Z$ factor.
} 
Let us write $\phi(z)$ in the form

$$
\phi(z)=-\log \left(\frac{z}{R_{U V}}\right)+h(z),
$$

where $h(z)$ has properties

$$
h(z)=O(z) \quad \text { for } z \rightarrow 0, \quad h(z)=\log \left(\frac{R_{I R}}{R_{U V}}\right)+O(1 / z) \quad \text { for } z \rightarrow \infty .
$$

By writing $\psi_{k}=e^{-\frac{1}{2} \phi(z)} f_{k}(z)$, Eq. (50) is converted into the Schrödinger equation

$$
f_{k}^{\prime \prime}-\frac{15}{4 z^{2}} f_{k}-k^{2} f_{k}-\frac{M_{U V}^{2} R_{U V}^{2}}{z^{2}} f_{k}=V(z) f_{k},
$$

where

$$
V(z)=\frac{3}{2} h^{\prime \prime}+\frac{9}{4}\left(h^{\prime}\right)^{2}-\frac{9}{2 z} h^{\prime}-\frac{M_{U V}^{2} R_{U V}^{2}}{z^{2}}\left(1-\frac{M^{2}(z)}{M_{U V}^{2}} e^{2 h(z)}\right) .
$$

Here

$$
V(z)=O(1 / z) \quad \text { for } z \rightarrow 0, \quad V(z)=\frac{\delta}{z^{2}}+O\left(1 / z^{3}\right) \text { for } z \rightarrow \infty,
$$

where $\delta=M_{I R}^{2} R_{I R}^{2}-M_{U V}^{2} R_{U V}^{2}$. The pure-AdS Eq. (20) - with $V(z)=0$ - has two independent solutions : $\sqrt{2 \pi k z} K_{\nu}(k z)$ and $\sqrt{2 \pi k z} I_{\nu}(k z)$. We are looking for the solution of the full equation defined by the boundary condition $\lim _{z \rightarrow \infty} e^{k z} f_{k}(z)=1$. Constructing an appropriate Green's function, we can write Eq. (20) (or Eq. (50) in the form of an integral equation. Let $\psi_{k}(z)=$ $e^{-\frac{3}{2} h(z)} \tilde{\psi}_{k}(z)$, then $\tilde{\psi}_{k}(z)$ is a solution of

$$
\tilde{\psi}_{k}(z)=\frac{k^{2} z^{2}}{2} K_{\nu}(k z)-z^{2} \int_{z}^{\infty} \frac{d \xi}{\xi} G(\xi, z ; k) V(\xi) \tilde{\psi}_{k}(\xi),
$$

where

$$
G(\xi, z ; k)=I_{\nu}(k z) K_{\nu}(k \xi)-I_{\nu}(k \xi) K_{\nu}(k z) .
$$

We normalize the solution $\tilde{\psi}_{k}(z)$ to 1 at $z=\epsilon$ :

$$
\Psi_{k}(z, \epsilon)=\frac{\psi_{k}(z)}{\psi_{k}(\epsilon)},
$$

then the two-point function is given by

$$
A\left(k^{2}\right)=\left[e^{3 \phi(z)} \frac{\partial_{z} \Psi_{k}(z, \epsilon)}{\Psi_{k}(z, \epsilon)}\right]_{\epsilon}^{\infty}=\left[e^{3 \phi(z)} \frac{\partial_{z} \tilde{\psi}_{k}(z)}{\tilde{\psi}_{k}(z)}-e^{3 \phi(z)} \frac{3}{2} h^{\prime}(z)\right]_{\epsilon}^{\infty} .
$$

All the $k$-dependence in Eq. (26) is contained in $\partial_{z} \log \left[\tilde{\psi}_{k}\right]$. It is therefore sufficient to compute $\partial_{z} \tilde{\psi}_{k}(z) / \tilde{\psi}_{k}(z)$. The solution of Eq. (23) is given by the series

$$
\tilde{\psi}_{k}(z)=\tilde{\psi}_{k}^{(0)}(z)+\tilde{\psi}_{k}^{(1)}(z)+\ldots,
$$


where

$$
\begin{gathered}
\tilde{\psi}_{k}^{(0)}(z)=\frac{k^{2} z^{2}}{2} K_{\nu}(k z), \\
\tilde{\psi}_{k}^{(n+1)}(z)=-\frac{k^{2} z^{2}}{2} \int_{z}^{\infty} \frac{d \xi}{\xi} G(\xi, z ; k) V(\xi) \tilde{\psi}_{k}^{(n)}(\xi) .
\end{gathered}
$$

It can also be written as

$$
\tilde{\psi}_{k}(z)=a(z, k) \frac{k^{2} z^{2}}{2} K_{\nu}(k z)+b(z, k) \frac{k^{2} z^{2}}{2} I_{\nu}(k z),
$$

where

$$
\begin{gathered}
a(z, k)=1+\frac{2}{k^{2}} \int_{z}^{\infty} \frac{d \xi}{\xi} V(\xi) I_{\nu}(k \xi) \tilde{\psi}_{k}(\xi), \\
b(z, k)=-\frac{2}{k^{2}} \int_{z}^{\infty} \frac{d \xi}{\xi} V(\xi) K_{\nu}(k \xi) \tilde{\psi}_{k}(\xi) .
\end{gathered}
$$

Using Eq. (27) we can write $a(z, k)=1+a_{1}+\ldots, b(z, k)=b_{1}+\ldots$, where

$$
\begin{aligned}
a_{1} & =\frac{1}{k} \int_{\mu}^{\infty} \frac{t}{k} V\left(\frac{t}{k}\right) I_{\nu}(t) K_{\nu}(t) d t, \\
b_{1} & =-\frac{1}{k} \int_{\mu}^{\infty} \frac{t}{k} V\left(\frac{t}{k}\right) K_{\nu}^{2}(t) d t,
\end{aligned}
$$

etc., and $\mu=k z$. Here, we are interested in the $\mu \ll 1$ case, since we want to study the region $k z \sim \epsilon \ll 1$.

The integrals are dominated by the contribution of the $t \sim \mu \ll 1$ region where we have $z V(z)=O(1)$ and, therefore,

$$
\begin{aligned}
& a_{1}=\frac{\mathcal{A}(\mu)}{k}+O\left(1 / k^{2}\right), \\
& b_{1}=-\frac{\mathcal{B}(\mu)}{k}+O\left(1 / k^{2}\right) .
\end{aligned}
$$

In general, we have $a_{n} \sim O\left(1 / k^{n}\right), b_{n} \sim O\left(1 / k^{n}\right)$. We arrive, therefore, at the standard Borntype series for $a$ and $b$.

Let us see now how the two-point function depends on $a$ and $b$. We have

$$
\frac{\partial_{z} \tilde{\psi}_{k}}{\tilde{\psi}_{k}}=\frac{\nu+2}{z}-\frac{k\left(K_{\nu+1}(k z)-r(k, z) I_{\nu+1}(k z)\right)}{K_{\nu}(k z)+r(k, z) I_{\nu}(k z)}+\frac{a^{\prime}}{a} \frac{K_{\nu}(k z)+\frac{b^{\prime}}{a^{\prime}} I_{\nu}(k z)}{K_{\nu}(k z)+r(k, z) I_{\nu}(k z)},
$$

where $r(k, z)=b(k, z) / a(k, z)$ and $a^{\prime}=\partial_{z} a, b^{\prime}=\partial_{z} b$. Let us consider massive and massless cases separately. 
Massive case, $\nu>2$

We have

$$
K_{\nu}(k z)+r(k, z) I_{\nu}(k z)=\frac{2^{\nu-1} \Gamma(\nu)}{(k z)^{\nu}}\left(1+\cdots-c_{\nu}(k, z)\left(\frac{k z}{2}\right)^{2 \nu} \frac{\Gamma(1-\nu)}{\Gamma(1+\nu)}+\cdots\right),
$$

where

$$
c_{\nu}(k, z)=1-\frac{2 r(k, z)}{\Gamma(\nu) \Gamma(1-\nu)}
$$

and

$$
K_{\nu+1}(k z)-r(k, z) I_{\nu+1}(k z)=\frac{2^{\nu} \Gamma(\nu+1)}{(k z)^{\nu+1}}\left(1+\cdots-c_{\nu+1}(k, z)\left(\frac{k z}{2}\right)^{2 \nu+2} \frac{\Gamma(-\nu)}{\Gamma(\nu+2)}+\cdots\right)
$$

SO

$$
\frac{\partial_{z} \tilde{\psi}_{k}}{\tilde{\psi}_{k}}=-\frac{2 \Gamma(1-\nu)}{z \Gamma(\nu)}\left(\frac{k z}{2}\right)^{2 \nu}+\frac{4 r(k, z)}{z \Gamma^{2}(\nu)}\left[1+\frac{z}{2 \nu} \frac{r^{\prime}(k, z)}{r(k, z)}\right]\left(\frac{k z}{2}\right)^{2 \nu}+\cdots,
$$

where $r^{\prime}(k, z)=\partial_{z} r(k, z)$ and $r(k, z)=b / a=O(1 / k)$, and dots represent higher powers of $k$. The $k$-dependent part of $A\left(k^{2}\right)$ is

$$
\begin{aligned}
A\left(k^{2}\right) & =R_{U V}^{3} e^{3 h(\epsilon)} \frac{2 \Gamma(1-\nu)}{\epsilon^{4} \Gamma(\nu)}\left(\frac{k \epsilon}{2}\right)^{2 \nu}\left(1+\frac{2 r}{\Gamma(\nu) \Gamma(1-\nu)}\left[1+\frac{\epsilon}{2 \nu} \frac{r^{\prime}}{r}\right]+O\left(1 / k^{2}\right)\right) \\
& +O\left((k \epsilon)^{2 \nu+n}\right),
\end{aligned}
$$

where $n>0$. The term proportional to $r(k, z)$ is the correction to the pure AdS result obtained in [2].

Massless case, $\nu=2$.

Here

$$
\frac{\partial_{z} \tilde{\psi}_{k}}{\tilde{\psi}_{k}}=-\frac{k\left(K_{3}(k z)-r I_{3}(k z)\right)}{K_{2}(k z)+r I_{2}(k z)}+\frac{a^{\prime}}{a} \frac{K_{2}(k z)+\frac{b^{\prime}}{a^{\prime}} I_{2}(k z)}{K_{2}(k z)+r I_{2}(k z)} .
$$

For $a^{\prime}=0, a=1, r=0$ this gives [2]

$$
\frac{\partial_{z} \tilde{\psi}_{k}}{\tilde{\psi}_{k}}=-\frac{k K_{3}(k z)}{K_{2}(k z)}=-\frac{z^{3} k^{4}}{4} \log k+O\left(k^{6} z^{5}\right) .
$$

For nonzero $r$,

$$
\frac{\partial_{z} \tilde{\psi}_{k}}{\tilde{\psi}_{k}}=-\frac{z^{3} k^{4}}{4} \log k\left(1+\frac{a^{\prime} z}{4 a}+O\left(k^{2} z^{2}, 1 / k^{2}\right)\right) .
$$

Here $a^{\prime} / a=O(1 / k)$. Therefore,

$$
A\left(k^{2}\right)=R_{U V}^{3} e^{3 h(\epsilon)} \frac{k^{4}}{4} \log k\left(1+\frac{a^{\prime} \epsilon}{4 a}+\cdots\right),
$$


to leading order in $k \epsilon$ and $1 / k$.

We can see, therefore, that in the $k^{2} \rightarrow \infty$ limit the two-point function can be written as

$$
A\left(k^{2}\right)=\tilde{Z}^{2}(\epsilon) A_{A d S}\left(k^{2}\right)(1+O(1 / k)),
$$

where $\tilde{Z}^{2}(\epsilon)=e^{3 h(\epsilon)}$ depends on interpolating metric, but not on $k$.

In summary, in this paper we have shown how Green's functions of composite operators in geometry duals of strongly coupled gauge theories can be computed in the IR and UV limit. We have found the pleasant result that the IR behavior of the Green's functions depends only on the "near-brane" geometry. We have also checked that, in all theories that are UV asymptotic to $\mathrm{N}=4$, 4-d super Yang-Mills, the UV Green's functions are universal.

It would be interesting to see if this result can be strenghtened to include the case when string corrections to classical gravity become important. It would be also important to see whether the formal similarity between Eq. (9) and the Callan-Symanzik equation is an accident, or it suggests instead a way of writing the C-S equation in geometry duals of gauge theories.

\section{Acknowledgements}

M.P. is supported in part by NSF grant no. PHY-9722083.

\section{References}

[1] J. Maldacena, Adv. Theor. Math. Phys. 2 (1998) 231, hep-th/9711200.

[2] S.S. Gubser, I.R. Klebanov and A.M. Polyakov, Phys.Lett. B428 (1998) 105, hepth/9802109.

[3] E. Witten, Adv. Theor. Math. Phys. 2 (1998) 253, hep-th/9802150.

[4] A. Kehagias and K. Sfetsos, hep-th/9902125.

[5] J.A. Minahan, hep-th/9902074.

[6] S.S. Gubser, hep-th/9902155.

[7] I.R. Klebanov and E. Witten, Nucl. Phys. B536 (1998) 199, hep-th/9807080.

[8] L. Girardello, M. Petrini, M. Porrati and A. Zaffaroni, hep-th/9810126.

[9] J.Distler and F.Zamora, hep-th/9810206.

[10] M. Günaydin, L.J. Romans and N.P. Warner, Phys. Lett. B154 (1985) 268; M. Pernici, K. Pilch and P. van Nieuwenhuizen, Nucl. Phys. B259 (1985) 460. 
[11] M. Günaydin, L.J. Romans and N.P. Warner, Nucl. Phys. B272 (1986) 598.

[12] L. Susskind and E. Witten, hep-th9805114.

[13] A. Peet and J. Polchinski, hep-th/9809022.

[14] C. Fronsdal, S. Ferrara and A. Zaffaroni, Nucl. Phys. B532 (1998) 153, hep-th/9802203.

[15] V.I. Arnol'd, Ordinary Differential Equations, (Springer-Verlag, Berlin, Heidelberg, New York, 1992). 